\title{
TRATAMIENTO EN LA PRENSA DIGITAL DE UN FENÓMENO GEOGRÁFICO: LA TURISTIFICACIÓN
}

\author{
Carlos Javier Egio Rubio* \\ Universidad de Murcia \\ https://orcid.org/0000-0002-7739-9051 \\ Piedad Fernández Toledo** \\ Universidad de Murcia \\ https://orcid.org/0000-0003-2036-1690
}

\section{RESUMEN}

La generalización del turismo barato -lowcost- y el uso masivo de plataformas colaborativas para el alquiler temporal de viviendas han potenciado en los últimos años los impactos que esta actividad económica ya de por sí tenía en ciudades de todo el mundo. Aunque el efecto más estudiado, por lo extendido, es la influencia directa en el precio del alquiler, la literatura especializada relaciona cada vez más aspectos como los conflictos asociados a la convivencia, la gentrificación, la privatización del espacio público o los problemas ambientales con un desarrollo turístico masivo y no planificado. Este artículo hace un análisis del seguimiento y tratamiento que se hace del término "turistificación" en la prensa española en el año 2018, como una aproximación al grado de instalación social del fenómeno.

Palabras clave: Turistificación; prensa, desarrollo turístico, análisis de discurso y géneros; medios de comunicación.

Coverage by the Spanish digital press of a geographic issue: touristification

\section{ABSTRACT}

The spreading of low cost tourism and the mass use of collaborative platforms for temporary rental of living accommodation in the last years have increased the social impact of these economic practices in many cities worldwide. Notwithstanding their direct influence on rental prices as the most direct effect and the focus of most research thus far, many studies are increasingly relating this massive and unplanned touristic development to issues such as

Fecha de recepción: 30 abril de 2019

Fecha de aceptación: 18 de noviembre de 2019

* Departamento de Geografía. Universidad de Murcia. Campus de La Merced. 30001 MURCIA (España). E-mail: cj.egiorubio@um.es

** Departamento de Filología Inglesa. Universidad de Murcia. Campus de La Merced. 30001 MURCIA (España).E-mail: piedad@um.es 
neighborhood conflicts, gentrification, public space privatizing or environmental problems. The present study tracks the use of the term "turistificación" (touristification) in the Spanish press during 2018, using a Discourse and Genre approach to examineits coverage in four newspapers, in order to ascertain its relative presence and its pervasivenessas a social issue.

Keywords: Touristification; press; touristic development, discourse and genre analysis.

\section{INTRODUCCIÓN}

En los últimos tres años cada vez es más frecuente encontrar en los medios de comunicación términos relacionados con el impacto social del turismo. Conceptos como "turistización", "turistificación" o "turismofobia" son cada vez más conocidos y acompañan, en el caso de España, a las ya clásicas informaciones sobre el aumento o disminución del número de visitantes extranjeros o sobre el peso del turismo en la economía. Mediante la investigación de la que se desprende este artículo pretendemos realizar una aproximación al interés que despiertan en la sociedad española los impactos de la actividad turística mediante un análisis del seguimiento que se hace de esta realidad en la prensa nacional, en su condición de catalizadora y difusora de aquello que afecta a nuestras vidas.

La turistización, o "turistificación" en un término más coloquial, es un fenómeno que afecta especialmente a grandes capitales y, en general, a ciudades importantes desde un punto de vista turístico a nivel internacional (Gravari-Barbas y Guinand, 2017). En el caso de Europa, por ejemplo, existen estudios sobre su impacto en urbes como Copenhague (Stors y Kagermeier, 2013), París (Freytag y Bouder, 2018), Berlín (Stores y Baltes, 2018) o Lisboa (Mendes, 2018), entre otras. Y dentro de la geografía nacional, más concretamente, van apareciendo tímidamente trabajos sobre la situación en ciudades como Valencia (Del Romero Renau, 2018) o Barcelona (Gravary-Barbas y Guinand, 2017).

La mayoría de estos trabajos reflejan, desde enfoques multidisciplinares, realidades similares en distintas partes del globo, que son producto de la confluencia de intereses encontrados (por un lado, el turismo barato y la irrupción de nuevas plataformas de "economía compartida" como AirBnB o Uber; por otro lado, los de las administraciones y la ciudadanía).

Del Romero Renau (2018: 2) define "turistificación” como "el proceso y el estado resultante de un desarrollo turístico masivo, relativamente espontáneo y no planificado, en un área determinada, que lleva a la propia transformación de dicha área en un recurso turístico" (traducido). El mismo autor incluye al turismo como uno de los cuatro regímenes de producto-consumo que están resultando afectados por las "economías de plataforma o compartidas", junto con la movilidad, el empleo y los residuos.

\subsection{La turistificación en España. ¿Un fenómeno de impacto creciente?}

Una de las consecuencias planetarias de la crisis económica de 2008 fue el cambio que se produjo en las estrategias de acumulación de capitales. Una vez explotada la burbuja 
del negocio inmobiliario tras la crisis de las hipotecas subprime, los grandes inversores centraron su atención en la industria turística. De este modo, tras una leve bajada, ya en 2012 se alcanzaba la cifra récord de mil millones de turistas (Murray Mas, 2014: 87). España, como uno de los polos de atracción de visitantes más importante del mundo, no escapa a esta realidad global, adaptada a las casuísticas de sus ciudades.

Si bien no se puede decir que el impacto de la industria turística en las ciudades españolas sea un fenómeno nuevo, puesto que hace décadas que la mayoría de estas se han adaptado a las necesidades de los visitantes mediante actuaciones de mayor o menor calado, algunas de las cuales han configurado por completo su fisonomía -es el caso de Bilbao y Barcelona, que han buscado en esta actividad económica un reemplazo para la práctica desaparición del sector industrial-, la generalización del turismo barato -lowcosty el uso masivo de las plataformas -en un principio "colaborativas"- para el alquiler temporal de viviendas, como la anteriormente mencionada Airbnb, han traído consigo nuevas consecuencias que escapan al control normativo existente. Tanto es así que "acudiendo al Plan Nacional e Integral de Turismo 2012-2015, no existe referencia al efecto invasivo del turismo, aunque se pueden encontrar diferentes iniciativas centradas en la potenciación del turismo urbano" (Guillén Navarro, 2018: 518).

En relación con todo lo descrito anteriormente, el efecto más estudiado, por lo extendido, es la influencia directa en el precio del alquiler de la sustitución de viviendas de uso residencial por viviendas de uso turístico. La aparición de herramientas colaborativas, utilizadas por los grandes fondos de inversión, multiplica los procesos de turistificación ya en marcha y alimenta otros como la gentrificación, "de manera que no se puede hablar de turistificación sin hablar de crisis de la vivienda" (Gil y Sequera, 2018: 24). En su obra Tourism and Gentrification in Contemporary Metropolises, Maria Gravari-Barbas y Sandra Guinand (2017) ya recogen la relación entre estos y otros conceptos -como el desarrollo de nuevas culturas urbanas-, con contribuciones sobre estrategias adoptadas en ciudades como Cartagena de Indias, Roma o Barcelona, para hacer frente a estas nuevas realidades y tensiones. Desde su perspectiva multidisciplinar en el análisis de doce casos -de las correspondientes doce ciudades-, las autoras hacen ver que en este nuevo panorama "no es oro todo lo que reluce" y que las políticas han de contemplar tanto a quien se beneficia como a las personas que resultan perjudicadas por los cambios en el entorno urbano.

Si nos centramos en lo que sucede en España, en la ciudad de Madrid solo un 5\% de las viviendas ofrecidas a través de Airbnb pueden asociarse a una economía colaborativa real, perteneciendo el resto actores comerciales y especializados que responden a "formas de economía tradicional basadas en la obtención de rentas inmobiliarias" (Gil y Sequera, 2018: 22). En el caso de la ciudad de Palma, se ha podido observar la relación directa entre turistización, revalorización y sustitución de inquilinos, de manera que la turistización se convierte en un factor fundamental "en la generación de desplazamientos, en este caso desahucios" (Vives-Miró y Rullán, 2017: 69).

Pero los efectos de un turismo masivo gestionado de una manera inadecuada van más allá de la subida de precios del alquiler. Acudiendo a la cada vez más extensa literatura especializada, entre los impactos negativos de la turistificación podrían destacarse: “ $a$ ) el aumento de los precios en los mercados inmobiliarios locales, provocando un creciente desplazamiento espacial de los residentes; $b$ ) el despojo material y simbólico de los residentes; $c$ ) la cre- 
ciente marginación de ciertas presencias, prácticas y consumos a través de políticas de que garanticen la seguridad-mediante vigilancia y control policial- de la gestión urbana; $d$ ) la promoción de nuevas formas de actividades de ocio hedonista y nocturno mercantilizado, que pueden ejercer presión sobre la habitabilidad de la comunidad; e) la desaparición del comercio minorista tradicional, que es sustituido por comercio elitista o "gurmetizado" dirigido al turismo" (Cabrerizo y otros, 2017: 78). A estos, la Red SET, de ciudades y regiones del Sur de Europa contra la turistización, une otros como la masificación en calles y plazas, la saturación de la red pública de transporte, la precarización de las condiciones laborales, el incremento de la contaminación, el uso desmesurado y la ampliación constante de infraestructuras o la banalización (tematización) de los entornos tanto urbanos como naturales ${ }^{1}$.

\subsection{El papel de los medios de comunicación en la repercusión de la turistificación}

Como indicábamos al principio, la presencia de la turistificación en los medios y el tratamiento que esta reciba puede ser buen indicador de la repercusión del fenómeno en España y sus grandes urbes, y sobre todo de la reacción por parte de los diferentes actores que conforman la sociedad. Los medios muestran las políticas públicas y las movilizaciones ciudadanas, y a su vez el tratamiento y la repercusión que tienen estas en los medios influyen directamente en las representaciones sociales de los aconteceres y la posterior la orientación de las políticas públicas. Como afirmara Sosa (2000: p. 14), "la noticia no es lo que pasa, sino un artefacto semiótico que representa simbólicamente lo que pasa", de ahí la importancia del análisis de los géneros periodísticos sobre asuntos como el que estamos tratando.

El análisis crítico del discurso (Van Dijk, 2010), al igual que el de los géneros discursivos (Bhatia, 2004, 2015), constituye un punto de partida eficaz, desde el que indagar en el comportamiento del lenguaje como reflejo del comportamiento social. En el caso de la turistificación, hay una serie de parámetros que nos permiten detectar el tratamiento dado por la prensa al concepto ligado al término, y que tienen que ver con las convenciones discursivas largamente asentadas en nuestra sociedad.

El género, tal y como lo describe John Swales (1990) en su ya clásica obra, es la unidad de comunicación reconocible por la comunidad discursiva que la utiliza con un objetivo comunicativo -o varios- y que tiene una composición formal convencional en lo referente a su estructuración, léxico, etc., que permiten su identificación y le aportan coherencia en el contexto de dicha comunidad. En cada discurso hay géneros más centrales -como la noticia en el discurso informativo - y otros más híbridos -caso de la columna, que respondiendo al objetivo de "creación de opinión" típico de este ámbito, obedece a patrones menos estrictos y tiene un componente más literario y personal, de manera que sus límites formales más claros son los del espacio disponible en la página -.

La división en subgéneros que establece Bhatia (2004) se basa en las tres dimensiones que propusiera Halliday (1985) de "campo" (field), "tono" (tenor) y "modo" (mode),

1 http://www.albasud.org/noticia/es/1027/manifiesto-fundacional-de-la-red-set-de-ciudades-del-sur-deeuropa-ante-la-turistizaci-n 
y permite establecer variaciones convencionales que responden a dichas dimensiones. En el caso de la información, el modo va estrechamente ligado al canal de comunicación -prensa escrita, radio, audiovisual-; el tono se relaciona con el grado de familiaridad entre hablantes -en este caso, de informalidad-formalidad- y el campo (lo que más nos concierne para este estudio), con el tema o ámbito de acción -actualidad, deportes, política, economía...-.

De esta manera, el hecho de que partamos de ciertos prototipos y convenciones permite, igualmente, establecer jerarquías y prioridades en cada ámbito discursivo, tanto en lo tocante a la elección de los propios géneros (por ej., noticia, editorial, columna, etc. en el caso de la prensa tradicional), como en la vinculación de un hecho noticiable a una sección determinada (lo que automáticamente lo liga a un campo, o área temática, de preferencia). No en vano, una de las funciones de los medios de comunicación es diferenciar aquellos acontecimientos que se consideran noticiosos de los que no y "catalogarlos". Es decir, los medios necesariamente llevan a cabo un filtrado para escoger aquellos hechos noticiables que consideran más apropiados para su propósito informativo, lo que finalmente depende de una línea editorial asociada a intereses políticos o económicos. Así, lo mismo que una obra académica se puede catalogar a menudo por distintas materias, en el caso del género "noticia", su asignación a un subgénero temático (ej., noticia de deportes, o de economía) llevará directamente a una valoración del hecho como más o menos trascendental según en qué sección se incluya y la posición y el espacio que se le otorgue dentro de dicha sección.

Igualmente, y bajo estas premisas, el hecho de que un tema se trate como tema opinable, noticiable -o ambas cosas -, así como la frecuencia y regularidad con que se mantenga a través del tiempo, nos puede dar una idea de su presencia relativa en la sociedad, por la condición de "eco" que tiene la prensa, marcando la actualidad y sus derivas. El que se escoja un género periodístico u otro ya permite acercarse a la intención discursiva de quien emite la información. Los géneros informativos (noticia y reportaje objetivo) son predominantemente narrativos y descriptivos; los géneros interpretativos (crónica y reportaje en profundidad) son explicativos, mientras que los géneros de opinión son básicamente argumentativos (Gutiérrez Vidrio, 2010: 174).

Concretamente, y en relación con el concepto que nos ocupa -turistificación -, los siguientes serán indicios del grado de "instalación" del tema a nivel social y de la percepción que de tal concepto se tiene (sin olvidar el papel de los medios en la propagación, ante lo cual estableceremos las comparaciones oportunas, en su caso):

- presencia del término "turistificación" en los titulares de distintas secciones, subsecciones y páginas de la prensa escrita, en sus ediciones digitales;

- frecuencia de aparición junto con otras temáticas o conceptos;

- caracterización del espacio geográfico asociado a la información;

- uso de fuentes por parte del medio para el tratamiento del fenómeno. 


\section{OBJETIVOS}

Decidimos abordar el presente estudio para confirmar nuestra percepción sobre la presencia creciente del término "turistificación" en la prensa escrita nacional, ante la escasez de trabajos que arrojen luz sobre el tema en el caso de nuestra Geografía.

El objetivo es comprobar la presencia de este fenómeno y, más específicamente:

- La frecuencia y regularidad de aparición del término (“occurrence");

- Su visibilidad relativa e impacto potencial según la presencia en géneros determinados (editorial, noticia, columna, ...) y secciones de la prensa nacional en las que se incluye preferentemente (local, nacional, viajes, etc.);

- Su ámbito geográfico, en relación con lo anterior: la información ¿se centra en lo local?; ¿en lo regional?; ¿específicamente engrandes ciudades?; ¿se trata más bien como un fenómeno global?

- Su impacto social según los temas y protagonistas con los que el término aparece relacionado;

- Las fuentes a las que han recurrido los medios, que marcan qué actores sociales están situando el fenómeno estudiado en la agenda informativa.

\section{METODOLOGÍA}

\subsection{Muestra}

Para el presente estudio nos centramos, por un lado, El País, El Mundo y ABC, como los tres diarios generalistas de alcance nacional con un mayor número de lectores en 2018 (papel + visor digital), según la Asociación para la Investigación de los Medios de Comunicación (AIMC, 2019: 55). A estos hemos sumado eldiario.es, el cuarto medio digital más consultado ese año, como se desprende del Digital News Report del Instituto Reuters (2018: 102), para poder comparar el tratamiento en dos periódicos de una línea editorial conservadora (El Mundo y ABC) y otros dos (El País y eldiario.es) caracterizados por tener una línea autodefinida como progresista. Por facilidad de acceso, recurrimos a las versiones digitales para todos los casos.

Utilizamos los buscadores de las páginas web de estos diarios ${ }^{2}$ para seleccionar títulos y subtítulos de piezas informativas publicadas en el transcurso de 2018 (el año siguiente a la irrupción de las principales movilizaciones relacionadas con el tema) que incluyeran el término "turistificación". Se eligió este vocablo y no "turistización", al estar el primero mucho más extendido en el ámbito periodístico, tal y como mostró una primera búsqueda tentativa.

Es importante señalar que se ha descartado incluir el término "turismofobia" en esta investigación, ampliamente utilizado por El Mundo y $A B C$, porque investigaciones previas consideran que su uso implica un sesgo ideológico, "criminalizador":

2 El funcionamiento de los motores de búsqueda no coincide al realizar la búsqueda, siendo ésta por palabras para eldiario.es y ABC, y por etiquetas en el caso de El País y El Mundo. 
Así pues, en turismofobia se atisba una muestra discursiva de ideología o creencias compartidas conducente (Van Dijk 2008: 208) a sostener los intereses del grupo socio profesional que se enriquece con los beneficios de la explotación de los recursos del turismo y pretende descalificar con esta etiqueta a los vecinos que se sienten perjudicados y plantean una gestión turística más racional y menos masificada. De este modo, la reivindicación se convierte en aversión, en acto vandálico o en extremista de izquierdas. Y, ante esta etiqueta cabe por parte del colectivo de vecinos o por parte de los mediadores (escritores, periodistas, etc.) su rechazo directo, ya que su redefinición como oposición a un tipo de turismo masificado mantiene inevitablemente el componente esencial de 'aversión al turismo' (Sanmatín Sáez, 2019: 19).

Siendo esta selección léxica una opción que ya desmarca a una prensa frente a otra a la hora de mostrar el posicionamiento ante un fenómeno social, hemos preferido descartarla por obvia, y nos hemos centrado en el uso de "turistificación" como término neutro sobre el que poder establecer comparaciones de uso.

La muestra resultante se compone de 168 artículos, de los cuales 97 se extrajeron del diario El País, 12 corresponden al diario El Mundo, 7 a $A B C$ y 52 a eldiario.es. Estas cifras denotan, de entrada, la importancia relativa otorgada al término y el concepto a que se refiere en los distintos medios durante el año 2018.

\subsection{Variables}

Hemos seleccionado aquellas variables que pueden aportar un grado importante de información, tanto sobre el fenómeno socio-geográfico a estudiar, como sobre el estatus otorgado por los medios al "hecho noticiable". De este modo, consideramos que aspectos como la frecuencia global del término en cada medio, la sección en la que se decide ubicar la información y el género discursivo utilizado, el ámbito geográfico objeto de atención -local, regional, nacional o internacional -, o las fuentes escogidas, nos hablan de la posición de los diferentes medios y su línea editorial ante la "turistificación". La tabla 1 presenta los aspectos mencionados y otros que complementan el conjunto de variables a analizar para llegar al objetivo propuesto.

\section{Tabla 1}

\section{VARIABLES}

\begin{tabular}{|l|}
\hline Términos y expresiones fraseológicas más frecuentes \\
\hline Sección \\
\hline Géneros discursivos \\
\hline Temas \\
\hline Fuentes \\
\hline Ámbito geográfico \\
\hline
\end{tabular}




\section{RESULTADOS E INTERPRETACIÓN}

\subsection{Términos y expresiones fraseológicas más frecuentes en los titulares}

Sin ser exhaustiva, la tabla 2 pretende recoger una comparativa que, debido al escaso número de titulares en dos de los medios, resulta difícil a priori. Así, en el caso de $A B C$ la frecuencia mayor de aparición de un término es 3, mientras que, en el extremo opuesto, El País presenta mucha más cantidad (hasta 50 repeticiones de la frase clave "pisos turísticos"). También es mucho mayor la variedad de palabras utilizadas en este segundo medio.

$\mathrm{Si}$ agrupamos todos los términos aparecidos en los titulares de los cuatro diarios, teniendo en cuenta que las similitudes semánticas permiten agrupar conceptos, los términos y expresiones más frecuentes son "pisos turísticos" o "viviendas turísticas"(65), “Airbnb" (31), "Madrid" (21), "Ayuntamiento" (13) y "turistificación" (9).

Tanto en la totalidad de la muestra como en el caso de El País, la segunda expresión más abundante es "Airbnb", plataforma objeto de controversia y directamente ligada al alquiler de viviendas turísticas tanto en España como en el resto del mundo. Solamente en eldiario.es "vecinos" está entre los términos más utilizados, ocupando en este caso el primer lugar.

"Madrid" es otro de los términos clave en los cuatro medios, lo que denota que el fenómeno estudiado formaba parte de la agenda informativa de la capital en 2018; de hecho fue una de las primeras ciudades - junto con Barcelona- en las que la administración local tuvo que tomar medidas de manera tajante (no en vano "Ayuntamiento" es la cuarta expresión más frecuente).

Esta primera aproximación ya nos muestra alguna de las tendencias que se seguirán encontrando en el resto de apartados: la turistificación es tratada desde un enfoque local, asociada fundamentalmente al impacto que tiene el auge de los pisos turísticos, propiciados por plataformas como Airbnb, en el precio de los alquileres. Son por tanto las administraciones locales las primeras en reaccionar.

El escaso número de artículos en $E l$ Mundo y $A B C$ no quiere decir que estos medios no registren los acontecimientos relacionados con el fenómeno estudiado, más bien corrobora que el tratamiento dado tiene un sesgo ideológico asociado al término "turismofobia", que es el que suelen utilizar con mayor frecuencia.

Tabla 2

TÉRMINOS Y EXPRESIONES MÁS FRECUENTES

\begin{tabular}{|l|l|l|l|}
\hline \multicolumn{1}{|c|}{ El País } & \multicolumn{1}{c|}{ eldiario.es } & \multicolumn{1}{c|}{ EI Mundo } & \multicolumn{1}{c|}{ ABC } \\
\hline 50 pisosturísticos & 9 vecinos & 3 Ayuntamiento & 3 Turistificación \\
\hline 24 Airbnb & 8 pisos turísticos & 3 viviendas & 3 Madrid \\
\hline 9 Madrid & 7 Airbnb & 3 centro & \\
\hline 7 viviendas turísticas & 7 Madrid & 3 Madrid & \\
\hline 7 alquiler turístico & 7 ciudad & & \\
\hline 6 Ayuntamiento & 6 turistificación & & \\
\hline
\end{tabular}




\begin{tabular}{|l|l|l|l|}
\hline \multicolumn{1}{|c|}{ El País } & \multicolumn{1}{c|}{ eldiario.es } & EI Mundo & ABC \\
\hline 6 Barcelona & 6 alquiler & & \\
\hline 6 turistas & 6 centro & & \\
\hline 6 ciudad(-es) & 5 vivienda & & \\
\hline 5 guerra & 5 Gobierno & & \\
\hline 4 turismo & 4 alquileres & & \\
\hline 4 vivienda & 4 ciudades & & \\
\hline 3 Comunidad & 4 turismo & & \\
\hline 3 piso & 4 barrio & & \\
\hline
\end{tabular}

\subsection{Secciones en que aparecen noticias sobre "turistificación"}

En la tabla 3 se muestra la presencia del término por secciones. Desde un estudio global destaca que la sección "Local" sea con diferencia la que más noticias relacionadas con la turistificación alberga (61,3\%), seguida de "Opinión" (15,5\%) y "Economía" (10,7\%). El confinamiento en una sección "Local" de un fenómeno de claras raíces globales puede indicar que, de momento, las consecuencias son más visibles que las causas del problema. En este mismo sentido pudo también influir la ausencia de una estrategia comunicativa común entre los colectivos vecinales que empezaron a denunciar algunos de los efectos de una inadecuada gestión turística, lo que contrasta con los encuentros estatales, internacionales y la jornada de protesta coordinada que tuvieron lugar en 2018.

En todo caso, tras este análisis general se esconde un tratamiento muy diferenciado entre los cuatro medios estudiados. Así, en el caso de El País, los artículos de opinión ocupan un segundo lugar, lo que indica la inclusión de un componente analítico -argumentativo y no solamente narrativo- en el tratamiento del fenómeno. Además, este es el único medio en el que el término aparece en muy diversas secciones, aunque tímidamente, lo que va anunciando que empieza a vislumbrarse su naturaleza compleja y multidisciplinar.

En contraste con estos datos, los escasos titulares en $\mathrm{El}$ Mundo y $A B C$ sobre el tema quedan confinados en la sección de información local exclusivamente, mientras que en eldiario.es "Economía" incluye la tercera parte de los titulares alojados en "Local", con muchas menos contribuciones críticas $(n=3)$, o, lo que es lo mismo, la turistificación es tratada como un fenómeno con un trasfondo económico -más que en el caso de El País - , pero sin que llegue a hacerse un análisis en profundidad desde esta perspectiva.

Tabla 3

SECCIONES

\begin{tabular}{|l|c|c|c|c|c|c|}
\hline SECCIONES & El País & El Mundo & ABC & eldiario.es & TOTAL & \% \\
\hline Local & 51 & 10 & 7 & 35 & 103 & $61,3 \%$ \\
\hline Opinión & 23 & 0 & 0 & 3 & 26 & $15,5 \%$ \\
\hline Economía & 5 & 0 & 0 & 13 & 18 & $10,7 \%$ \\
\hline Tecnología & 5 & 0 & 0 & 0 & 5 & $3,0 \%$ \\
\hline
\end{tabular}




\begin{tabular}{|l|c|c|c|c|c|c|}
\hline SECCIONES & EI País & EI Mundo & ABC & eldiario.es & TOTAL & $\%$ \\
\hline Viajes & 4 & 0 & 0 & 0 & 4 & $2,4 \%$ \\
\hline $\begin{array}{l}\text { Ciencia y medio ambiente } \\
\text { (Planeta Futuro) }\end{array}$ & 1 & 1 & 0 & 0 & 2 & $1,2 \%$ \\
\hline Turismo & 1 & 1 & 0 & 0 & 2 & $1,2 \%$ \\
\hline Tendencias & 2 & 0 & 0 & 0 & 2 & $1,2 \%$ \\
\hline Internacional & 1 & 0 & 0 & 0 & 1 & $0,6 \%$ \\
\hline Editorial & 1 & 0 & 0 & 0 & 1 & $0,6 \%$ \\
\hline Cultura & 1 & 0 & 0 & 0 & 1 & $0,6 \%$ \\
\hline Vídeos & 1 & 0 & 0 & 0 & 1 & $0,6 \%$ \\
\hline Política & 1 & 0 & 0 & 1 & 2 & $1,2 \%$ \\
\hline & 97 & 12 & 7 & 52 & 168 & \\
\hline
\end{tabular}

\subsection{Géneros discursivos y status de la noticia o tema}

En coherencia con el apartado anterior, también diferenciada es la distribución de géneros periodísticos -o discursivos -, como se aprecia en la tabla 4.

Los cuatro editoriales, que como tales deben marcar la línea ideológica del periódico, y la profusión de noticiasen El País dejan claro que el medio tiene una postura favorable a la regulación de los pisos turísticos. Aparece una y otra vez a lo largo de 2018 la consecuencia más evidente: la subida de precios de los pisos de alquiler. Con veinte artículos de opinión, así como siete entrevistas y doce reportajes, la variedad de géneros en este medio permite un tratamiento plural y en profundidad de un fenómeno geográfico complejo como este.

Respecto a eldiario.es la abundancia de piezas informativas tratadas como noticia, opinión, reportaje y entrevista, con la variedad de géneros que esto supone, permite un equilibrio entre lo puramente narrativo y una exposición más analítica y argumental.

Por último, El Mundo y $A B C$, solo presentan 14 noticias en total y dos reportajes en cada caso, lo que no supone una contribución de peso que permita ofrecer un análisis detallado. Esto se debe fundamentalmente a una exposición de algunos efectos asociados a la turistificación sin que se se establezca una relación de causalidad con el fenómeno estudiado.

Tabla 4

GÉNEROS PERIODÍSTICOS

\begin{tabular}{|l|c|c|c|c|c|c|}
\hline GÉNERO & EI País & EI Mundo & ABC & eldiario.es & TOTAL & \% \\
\hline Noticia & 53 & 10 & 4 & 27 & 94 & $56,0 \%$ \\
\hline Opinión & 20 & 0 & 1 & 9 & 30 & $17,9 \%$ \\
\hline Reportaje & 12 & 2 & 2 & 11 & 27 & $16,1 \%$ \\
\hline
\end{tabular}




\begin{tabular}{|l|c|c|c|c|c|c|}
\hline GÉNERO & El País & El Mundo & ABC & eldiario.es & TOTAL & \% \\
\hline Entrevista & 7 & 0 & 0 & 5 & 12 & $7,1 \%$ \\
\hline Editorial & 4 & 0 & 0 & 0 & 4 & $2,4 \%$ \\
\hline Crónica & 1 & 0 & 0 & 0 & 1 & $0,6 \%$ \\
\hline & 97 & 12 & 7 & 52 & 168 & \\
\hline
\end{tabular}

\subsection{La regulación de las viviendas turísticas, el impacto más destacado}

Si bien la mayoría de los impactos asociados a la turistificación aparecen contemplados de una u otra manera en las piezas informativas analizadas, el encarecimiento de los alquileres destaca por encima de todos; recogido, además, en los artículos que desde diferentes géneros tratan la regulación de las viviendas turísticas $(38,5 \%)$ y el derecho a la vivienda $(18,8 \%)$, este se convierte en el tema principal de más de la mitad de las informaciones $(57,3 \%)$.

TABLA 5

\section{ASPECTOS RELACIONADOS CON LA TURISTIFICACIÓN}

\begin{tabular}{|l|c|c|c|c|c|c|}
\hline $\begin{array}{l}\text { ASPECTOS RELACIONADOS } \\
\text { CON "TURISTIFICACIÓN" }\end{array}$ & EI País & EI Mundo & ABC & eldiario.es & TOTAL & $\%$ \\
\hline Regulación viviendas turísticas & 71 & 10 & 5 & 39 & 125 & $38,5 \%$ \\
\hline Derecho a la vivienda & 34 & 2 & 4 & 21 & 61 & $18,8 \%$ \\
\hline Convivencia & 13 & 1 & 1 & 7 & 22 & $6,8 \%$ \\
\hline Turistificación/Gentrificación & 12 & 1 & 2 & 4 & 19 & $5,8 \%$ \\
\hline Privatización del espacio público & 6 & 1 & 0 & 11 & 18 & $5,5 \%$ \\
\hline Masificación/Servicios & 5 & 2 & 0 & 8 & 15 & $4,6 \%$ \\
\hline Medio ambiente & 8 & 1 & 0 & 6 & 15 & $4,6 \%$ \\
\hline Tematización & 5 & 0 & 0 & 6 & 11 & $3,4 \%$ \\
\hline Impacto en el pequeño comercio & 4 & 1 & 0 & 3 & 8 & $2,5 \%$ \\
\hline Precariedad laboral & 3 & 0 & 0 & 4 & 7 & $2,2 \%$ \\
\hline Impacto en el sector hotelero & 7 & 0 & 0 & 0 & 7 & $2,2 \%$ \\
\hline Turismofobia & 5 & 0 & 0 & 0 & 5 & $1,5 \%$ \\
\hline Centro/Periferia & 1 & 0 & 0 & 3 & 4 & $1,2 \%$ \\
\hline Mercantilización del ocio & 0 & 0 & 0 & 3 & 3 & $0,9 \%$ \\
\hline Pérdida del tejido social & 0 & 0 & 0 & 3 & 3 & $0,9 \%$ \\
\hline Problemas en la movilidad & 1 & 0 & 0 & 0 & 1 & $0,3 \%$ \\
\hline Amenazas al patrimonio & 0 & 0 & 0 & 1 & 1 & $0,3 \%$ \\
\hline
\end{tabular}


Los dos reportajes de $A B C$ y los dos de $E l M u n d o$ tienen un enfoque local, tres de estos centrados en Madrid y uno ( $E l$ Mundo) en las fallas de Valencia. En estos cuatro reportajes se tratan las reivindicaciones de la vecindad, mientras que las cuatro piezas informativas restantes se centran casi exclusivamente en la regulación de viviendas, coincidiendo con las medidas adoptadas durante ese año por las administraciones locales y regionales.

En El País y eldiario.es se aprecia un escenario más variado en cuanto a impactos y temáticas, reflejando las consecuencias asociadas al turismo masivo que recogen estudios geográficos y sociales desarrollados en otras latitudes (Stors y Kagermeier 2013; GravariBarbas y Guinand 2017; Freytag y Bouder 2018; Stores y Baltes 2018: Mendes 2018): problemas de convivencia, gentrificación, privatización del espacio público, masificación, problemas medioambientales, tematización, impacto en el pequeño comercio, precariedad laboral, mercantilización del ocio, pérdida del tejido social o impacto en el negocio hotelero. Destaca en todo caso, un mayor énfasis en eldiario.es en los aspectos negativos de la turistificación en el ámbito social y laboral.

\subsection{Fuentes utilizadas}

Si los acontecimientos narrados en las piezas informativas de los cuatro medios analizados se distribuyeran a lo largo de una línea de tiempo, rápidamente se podría comprobar que estamos ante un fenómeno en el que entran en juego fundamentalmente cuatro actores: los ayuntamientos de algunas de las ciudades más turísticas del país (Madrid, Barcelona, Valencia o Palma), las empresas que gestionan plataformas de alquiler de viviendas turísticas (fundamentalmente Airbnb), las plataformas vecinales que reclaman una regulación que evite una subida de precios del alquiler y, en un menor grado, las patronales del sector hotelero exigiendo esa misma regulación para disminuir la competencia.

En el año 2018 empiezan a gestarse y a aprobarse muchas de las medidas y normas autonómicas y locales que, resultantes de la presión vecinal de 2017, empezarán a poner coto al alquiler de viviendas con una función turística. De hecho, el mismo 4 de enero es noticia que la Generalitat de Cataluña recauda 4 millones en multas y que el Ayuntamiento de Madrid suspende la conversión de pisos en edificios turísticos en el centro de la ciudad, quizá como respuesta a la denuncia que en julio del año anterior interpusieron ante la justicia las asociaciones vecinales de Sol y Barrio de las Letras ante lo que consideraban una actitud pasiva del consistorio frente a la proliferación de viviendas de este tipo.

Si bien también cuentan con competencias que podrían facilitar la regulación del alquiler, las comunidades autónomas parecen ocupar un segundo plano en los medios y la intervención del Gobierno central solo aparece en diciembre, asociada a un decreto del alquiler que no contará finalmente con herramientas para intervenir en los precios.

Todo lo descrito puede adquirir un enfoque u otro dependiendo de la fuente que se utilice para darle forma. La tabla 6 recoge la variable que hemos utilizado para indagar en este aspecto. En un análisis global que incluya los cuatro diarios, la principal fuente de información la constituyen los colectivos vecinales $(26,6 \%)$, seguidos de los ayuntamientos $(15,6 \%)$. Es decir, la acción organizada de la ciudadanía, demandando fundamentalmente una limitación del arrendamiento ocasional que detuviera el alza de los precios, fue imprescindible para situar en la agenda mediática los problemas asociados a 
la turistificación. Es incluso muy probable que fuera esta presión vecinal recogida en los medios, que también fue importante en 2017, la que obligara a ayuntamientos como los de Madrid y Barcelona, cuyos equipos de gobierno decían haber llegado al poder gracias precisamente al apoyo de los colectivos ciudadanos, a tomar cartas en el asunto mediante el desarrollo de una nueva normativa.

Por otro lado puede destacarse que El País presenta el uso de fuentes más diverso y equilibrado, que incluye la consulta en muchos casos a expertos, universidades, asociaciones hoteleras y a las patronales y empresas de pisos turísticos, mientras que eldiario.es se decanta significativamente por los colectivos vecinales al hacerse eco de la información que estos generan y constituir esta el $48 \%$ de sus piezas informativas.

Tabla 6

FUENTES DE INFORMACIÓN

\begin{tabular}{|c|c|c|c|c|c|c|}
\hline FUENTES UTILIZADAS & El País & EI Mundo & $\mathbf{A B C}$ & eldiario.es & TOTAL & $\%$ \\
\hline Colectivosvecinales & 14 & 4 & 3 & 25 & 46 & $26,6 \%$ \\
\hline Ayuntamientos & 15 & 5 & 2 & 5 & 27 & $15,6 \%$ \\
\hline Universidad/Expertos & 12 & 0 & 0 & 8 & 20 & $11,6 \%$ \\
\hline $\begin{array}{l}\text { Asociaciones } \\
\text { empresariales hoteleras }\end{array}$ & 10 & 3 & 0 & 1 & 14 & $8,1 \%$ \\
\hline Comunidades Autónomas & 7 & 1 & 0 & 1 & 9 & $5,2 \%$ \\
\hline Autor/a del artículo & 11 & 0 & 0 & 3 & 14 & $8,1 \%$ \\
\hline $\begin{array}{l}\text { Patronal pisos turísticos y } \\
\text { empresas }\end{array}$ & 11 & 0 & 0 & 2 & 13 & $7,5 \%$ \\
\hline Partidos políticos & 3 & 0 & 0 & 3 & 6 & $3,5 \%$ \\
\hline Director del medio & 4 & 0 & 0 & 0 & 4 & $2,3 \%$ \\
\hline $\begin{array}{l}\text { CNMC (Comisión } \\
\text { Nacional del Mercado y la } \\
\text { Competencia) }\end{array}$ & 3 & 0 & 0 & 0 & 3 & $1,7 \%$ \\
\hline Revistas científicas & 0 & 1 & 0 & 1 & 2 & $1,2 \%$ \\
\hline $\begin{array}{l}\text { Estado/Gobierno/ } \\
\text { Ministerios }\end{array}$ & 2 & 0 & 0 & & 2 & $1,2 \%$ \\
\hline $\begin{array}{l}\text { Colegio Administradores } \\
\text { de Fincas }\end{array}$ & 1 & 0 & 0 & 0 & 1 & $0,6 \%$ \\
\hline Justicia & 1 & 0 & 0 & 0 & 1 & $0,6 \%$ \\
\hline Comerciantes & 1 & 0 & 0 & 0 & 1 & $0,6 \%$ \\
\hline Policía & 1 & 0 & 0 & 0 & 1 & $0,6 \%$ \\
\hline Vecinos (sin organizar) & 0 & 0 & 0 & 1 & 1 & $0,6 \%$ \\
\hline Sindicatos de clase & 0 & 0 & 0 & 1 & 1 & $0,6 \%$ \\
\hline
\end{tabular}




\begin{tabular}{|l|c|c|c|c|c|c|}
\hline FUENTES UTILIZADAS & EI País & EI Mundo & ABC & eldiario.es & TOTAL & \% \\
\hline ONU & 0 & 0 & 0 & 1 & 1 & $0,6 \%$ \\
\hline Otras fuentes (variadas) & 4 & 0 & 2 & 0 & 6 & $3,5 \%$ \\
\hline & 100 & 14 & 7 & 52 & 173 & $100,0 \%$ \\
\hline
\end{tabular}

\section{6. Ámbitos geográficos objeto de atención}

En las tablas 7.1 y 7.2 se corrobora el tratamiento eminentemente local del fenómeno. Esto se desprende tanto de la sección que ocupan las piezas informativas, en un $61,3 \%$ el apartado "Local", como del lugar al que hacen referencia, en un $46 \%$ ciudades. Seguirían en importancia el ámbito nacional (23\%), el autonómico (17\%) y el internacional (15\%).

TABLA 7.1.

\section{ÁMBITO GEOGRÁFICO OBJETO DE ATENCIÓN}

\begin{tabular}{|l|r|r|r|r|r|r|}
\hline & \multicolumn{1}{|c|}{ EI País } & \multicolumn{1}{|c|}{ EI Mundo } & \multicolumn{1}{|c|}{ ABC } & eldiario.es & \multicolumn{1}{c|}{ TOTAL } & \multicolumn{1}{c|}{ \% } \\
\hline Internacional & 21 & 1 & 0 & 3 & 25 & $15 \%$ \\
\hline Nacional & 21 & 1 & 1 & 15 & 38 & $23 \%$ \\
\hline Autonómico & 16 & 2 & 0 & 10 & 28 & $17 \%$ \\
\hline Local & 39 & 8 & 6 & 24 & 77 & $46 \%$ \\
\hline & 97 & 12 & 7 & 52 & 168 & $100 \%$ \\
\hline
\end{tabular}

Si nos circunscribimos al peso de las ciudades y comunidades autónomas en las informaciones aparecidas en los diarios estudiados, casi la mitad de las piezas se podrían ubicar en Madrid (45\%) y su comunidad autónoma (4\%), lo que puede tener que ver con la infraestructura con la que cuentan los medios nacionales en la capital del Estado. No obstante, quizá sea más significativo señalar que las tres grandes ciudades (Madrid, Barcelona y Valencia) agrupan el 65\% de las noticias aparecidas. A la vista de los resultados, cabe preguntarse si la información relacionada con la turistificación se concentra donde mayor desarrollo alcanza el fenómeno o bien donde están las redacciones de los diarios estudiados, puesto que incluso eldiario.es, con un modelo descentralizado, cuenta con una redacción considerablemente más grande y con un mayor número de profesionales en Madrid. Parece que esto podría influir en el hecho de que las grandes ciudades queden sobrerrepresentadas en las informaciones mientras que otras de un tamaño medio, en las que el turismo de masas es sin duda una realidad importante, como pudieran ser Alicante, Las Palmas o Córdoba, apenas reciben cobertura, con el impacto que eso supone en la percepción de la ciudadanía.

A su vez no puede despreciarse el efecto en la visibilidad de la turistificación en las grandes aglomeraciones urbanas que provoca la presencia de tejidos asociativos dinámicos que generen información con sus actuaciones. En todo caso, aunque en mucho menor grado, están también presentes en las informaciones otras ciudades y comunidades con un peso turístico importante como Andalucía, Canarias o Baleares. 
Tabla 7.2.

ÁMBITO GEOGRÁFICO OBJETO DE ATENCIÓN (POR CIUDADES Y COMUNIDADES AUTÓNOMAS)

\begin{tabular}{|l|r|r|r|r|r|r|}
\hline & \multicolumn{1}{|c|}{ El País } & El Mundo & ABC & eldiario.es & TOTAL & \% \\
\hline $\begin{array}{l}\text { Madrid } \\
\text { (Comunidad) }\end{array}$ & 3 & 1 & 0 & 0 & 4 & $4 \%$ \\
\hline Madrid (Ciudad) & 24 & 4 & 6 & 13 & 47 & $45 \%$ \\
\hline Cataluña & 4 & 0 & 0 & 0 & 4 & $4 \%$ \\
\hline Barcelona & 11 & 0 & 0 & 0 & 11 & $10 \%$ \\
\hline Andalucía & 3 & 1 & 0 & 1 & 5 & $5 \%$ \\
\hline Málaga & 0 & 0 & 0 & 3 & 3 & $3 \%$ \\
\hline Sevilla & 0 & 0 & 0 & 1 & 1 & $1 \%$ \\
\hline Valencia \\
(Comunidad) & 2 & 0 & 0 & 1 & 3 & $3 \%$ \\
\hline Valencia (Ciudad) & 1 & 4 & 0 & 5 & 10 & $10 \%$ \\
\hline Benidorm & 1 & 0 & 0 & 0 & 1 & $1 \%$ \\
\hline Canarias & 1 & 0 & 0 & 6 & 7 & $7 \%$ \\
\hline Gran Canarias & 0 & 0 & 0 & 1 & 1 & $1 \%$ \\
\hline Baleares & 2 & 0 & 0 & 0 & 2 & $2 \%$ \\
\hline Palma & 2 & 0 & 0 & 0 & 2 & $2 \%$ \\
\hline Galicia & 1 & 0 & 0 & 0 & 1 & $1 \%$ \\
\hline Euskadi & 0 & 0 & 0 & 1 & 1 & $1 \%$ \\
\hline Cantabria & 0 & 0 & 0 & 1 & 1 & $1 \%$ \\
\hline Mérida & 0 & 0 & 6 & 34 & 105 & $100 \%$ \\
\hline & & 0 & 0 & 1 & & $1 \%$ \\
\hline
\end{tabular}

El resultado de esta variable es coherente con lo hasta aquí expuesto. Aunque, si bien es lógico que lo local tenga más peso que lo regional en la información, puesto que los efectos de la turistificación tienen lugar en ciudades concretas, es preocupante que esto pueda influir en que la presión vecinal recaiga sobre administraciones que carecen de competencias para hacer frente por sí solas al fenómeno (Marqués, 2019). En todo caso, fueron noticia en 2018 los acontecimientos relacionados con la regulación de los pisos de alquiler turísticos que tuvieron lugar sobre todo en los consistorios, a lo que habría que sumar el que algunas comunidades autónomas como Cataluña, la Comunidad de Madrid, Baleares, Canarias y la Comunidad Valenciana comenzaran a desarrollar una regulación incipiente.

A su vez, la inestabilidad política, que llevó a una moción de censura entre el 31 de mayo y el 1 de junio, con el consiguiente cese del Gobierno, pudo ser determinante en 
que no fuera hasta diciembre de ese año que se anunciara un decreto sobre vivienda al que muchos colectivos sociales se opusieron por no incluir un índice de precios.

Por último, si nos centramos en un análisis por medios, El País muestra, no obstante, la trascendencia de la temática más allá de nuestras fronteras, con una proporción considerable de artículos de índole internacional $(21,6 \%)$, mientras que eldiario.es reparte la atención entre lo local (en primer lugar), lo autonómico y lo nacional, con un peso muy reducido en lo internacional $(5,8 \%)$. $A B C$ se ciñe casi exclusivamente a Madrid en su información, mientras que El Mundo reparte ésta entre Madrid y Valencia -lo cual lleva a recordar que el género preferente en ambos casos es la noticia, y casi exclusivamente se limitan a la difusión de las medidas tomadas por las administraciones. El tratamiento en profundidad en $A B C$ y El Mundo es muy secundario, por tanto, y no permite al conjunto de lectores reconocer un fenómeno geográfico complejo como es el de la turistificación.

\section{CONCLUSIONES}

En este trabajo hemos analizado la presencia y el grado de importancia relativa del concepto de "turistificación" en cuatro de los diarios nacionales en su versión digital, entendiendo que cómo esté representado e "instalado" en los medios puede influir en la percepción que se tenga de este fenómeno, y por tanto en la respuesta que se dé desde administraciones y colectivos sociales.

Los datos señalan, por un lado, un evidente sesgo a la hora de tratar el tema, que se muestra en la escasa presencia del término "turistificación" como tal en dos de los diarios ( $E l$ Mundo y $A B C$ ), abundando por el contrario una palabra con una profunda carga ideológica como es "turismofobia". Eso nos lleva a sugerir la necesidad de utilizar más de una fuente a la hora de recurrir a la prensa para documentarse sobre este y otros fenómenos sociogeográficos; por otro lado, se constata una tímida presencia de la turistificación en los medios de comunicación fuera de la esfera local, de manera que, al menos durante el año estudiado -2018-, apenas trascendía de dicho entorno ni era tratado de forma general como un asunto de agenda a niveles regional o estatal en la prensa analizada. Que el tratamiento mayoritario que se da a un fenómeno de causas y consecuencias globales como este quede reducido a su impacto en el ámbito más próximo debería motivar una reflexión sobre la manera en que se cubren desde los medios de comunicación realidades complejas que afectan de manera directa a las formas de vida de una parte importante de la sociedad. Ya sea por falta de medios o por el diferente grado de interés que despierta en cada cabecera dependiendo de las respectivas líneas editoriales, se echa en falta en este caso un tratamiento en profundidad a través de los géneros que lo permiten-caso del reportaje-, que facilite contrastar los hechos con lo que sucede incluso en otros países, de manera que quienes acuden a los medios para informarse puedan obtener algo más que eventos inconexos.

Puesto que hemos dicho que consideramos que la manera en que se refleja un determinado fenómeno en los medios tiene una repercusión sobre las representaciones sociales compartidas por la ciudadanía sobre este, y que esto a su vez puede influir en la posterior orientación de las políticas públicas, parece claro que, al menos en el año estudiado, el tratamiento dado en los medios no era el más adecuado para adquirir una comprensión 
global de los acontecimientos. Sin embargo, aunque este es el panorama general, debe diferenciarse entre la manera en que abordan este asunto los medios con una línea editorial con una tendencia progresista -El País, un periódico con una nutrida red de corresponsales, cuenta con casi un $21,6 \%$ de piezas informativas internacionales, mientras que un $28,8 \%$ de las noticias de eldiario.es son de alcance nacional-, de cómo lo hacen las otras dos cabeceras estudiadas, en las que las noticias locales ocupan un $66,6 \%$ y un $85,7 \%$ respectivamente.

Como se plantea en la sección anterior, cabe preguntarse además si el hecho de que las tres ciudades con un mayor número de habitantes concentren un $65 \%$ de las piezas informativas tiene que ver con la relevancia de la turistificación en estos lugares o con el modelo de gestión de los diarios, que cuentan con la mayor parte de la plantilla en estos lugares, dejando fuera del foco mediático otros núcleos en los que los problemas asociados a un turismo de masas descontrolado pueden ser mayores.

Sea como fuere, es bastante probable que, antes o después, las administraciones estatal e incluso europea (Marqués, 2019) tengan que adoptar medidas antes de que los efectos negativos de un turismo masivo no regulado alcancen dimensiones críticas en lo tocante a aspectos como la convivencia, movilidad, contaminación y otros tantos impactos, como los que aparecen de manera tímida todavía en las tablas analizadas.

Si bien no tenemos constancia de estudios sobre la turistificación y sus consecuencias para el territorio español en general -sí de aportaciones centradas en ciudades concretas -, el ejemplo de otras latitudes debería bastar para atender este fenómeno y sus consecuencias desde un punto de vista integral, velando por los intereses de todas las partes para intentar casarlos en lo que, previsiblemente, constituyan nuevos y más armoniosos modelos de ciudad y de barrios, e incluso de negocio turístico.

En este trabajo nos hemos centrado en el uso de un término para "tirar del hilo" y constatar la importancia que se le daba en los medios en 2018. Los resultados arrojan datos reveladores que sirven de índice para ahondar en aspectos determinados y comprobar su evolución en nuevos estudios. En esta primera incursión nos hemos limitado a tres periódicos en su versión digital y a otro nativo digital, pero se podría ampliar el abanico a otros canales -radio y televisión - e incluso a las redes sociales.

Es de esperar que en el año actual y sucesivos, el término que nos ha ocupado "salte" a secciones más allá de lo local y lo regional, y se haga más habitual y digno no solo de seguimiento, sino de un tratamiento más generalizado y contrastado. Puede arrojar más luz sobre el estudio académico de la turistificación, desde un enfoque como el aquí expuesto, la inclusión de conceptos como gentrificación o turismofobia para comparar su grado de instalación relativo, y cómo funcionan como índice de la presión social respecto a la regulación del turismo.

Igualmente, un enfoque sociocrítico, más cualitativo y centrado en el análisis del contenido, que aborde los discursos de los distintos actores -colectivos vecinales y ciudadanía, administración, turistas, empresas de alquiler turístico y de la hostelería, grupos ecologistas... -, contrastándolos entre sí y con el desarrollo de hechos y acontecimientos al respecto, permitiría identificar el grado de tensión y la deriva de un fenómeno para el que no parece haber soluciones claras y sí muchos recovecos legales e intereses económicos (Marqués, 2019). 


\section{BIBLIOGRAFÍA}

ARROYO ALBA, M. (2019): «Acceso a la vivienda en contextos de gentrificación y turistificación urbana», Tendencias Sociales. Revista de Sociología, no 4, p. 79-98

ASOCIACIÓN PARA LA INVESTIGACIÓN DE MEDIOS DE COMUNICACIÓN (2019): Marco general de los medios en España 2019. Madrid, AIMC.

BHATIA, V.J.K. (2004): Worlds of Written Discourse.A Genre-based View. Londres, Continuum.

BHATIA, V.J.K. (2015): «Critical genre analysis. Theoretical preliminaries». Hermes Journal of Language and Communication in Business, vol. 54, pp. 9-20.

CABRERIZO, C.; SEQUERA, J. y BACHILLER, P. G. (2016): «Entre la turistificación y los espacios de resistencia en el centro de Madrid: Algunas claves para (re) pensar la ciudad turística», Ecología Política, nº 52, p. 78-82.

DEL ROMERO RENAU, L. (2018): «Touristification, sharing economies and the new geography of urban conflicts», Urban Science, vol. 2 (4), pp. 2-17.

ESPEJO MARÍN, C. y LÓPEZ DE LOS MOZOS, Á. (1995): “La publicidad inmobiliaria. Fuente para el estudio del mercado de la vivienda", en VI Coloquio Ibérico de Geografía, Oporto, Universidad de Oporto, vol. I, pp. 115-121.

FREYTAG, T, y BAUDER, M. (2018): «Bottom-up touristification and urban transformations in Paris»,Tourism Geographies, vol. 20 (3), pp. 443-460.

GUTIÉRREZ VIDRIO, S. (2010): «Discurso periodístico: una propuesta analítica», Comunicación y Sociedad, $\mathrm{n}^{\circ} 14$, p. 169-198.

GIL, J. y SEQUERA, J. (2018): «Expansión de la ciudad turística y nuevas resistencias. El caso de Airbnb en Madrid», Empiria. Revista de Metodología de Ciencias Sociales, $\mathrm{n}^{\mathrm{o}} 41, \mathrm{p} .15-32$

GRAVARI-BARBAS, M. y SANDRA, G. (Eds.) (2017): Tourism and Gentrification in Contemporary Metropolises. International Perspectives. Londres, Routledge.

GUILLÉN NAVARRO, N.A. (2018): «Turismo y medio ambiente: la sostenibilidad urbana ante la turistificación», en Observatorio de Políticas Ambientales 2018. Madrid, CIEMAT, pp. 517-539.

HALLIDAY, M.A.K. (1991): Language, Context, and Text: Aspects of Language in a Social-Semiotic Perspective. Oxford/Geelong: OUP/ Deakin University Press.

MARQUÉS, R. (2019): «Overtourism, o cómo hacer frente a la sombra alargada del turismo de masas» en CN Traveler Newsletter, 23 de octubre de 2019. Disponible en https://www.traveler.es/viajeros/articulos/soluciones-overtourism-ciudades-europeas $/ 16449$

MARTÍNEZ MAURI, M. (2015): «Una mirada sobre la turistificación de la antropología del desarrollo en el Estado español», PASOS. Revista de Turismo y Patrimonio Cultural, vol. 13 (2), p. 347-358.

MENDES, L. (2018): "The Panacea of touristification as a scenario of post-capitalist crisis", en Crisis, Austerity and Transformation. How Disciplinary Neoliberalism is Changing Portugal. Londres, Lexington Books, pp. 25-46.

MURRAY MÁS, I. (2014) «Bienvenidos a la fiesta: turistización planetaria y ciudadesespectáculo (y algo más)», Ecología Política, nº 47, pp. 87-91. 
QUINTANA, B. ET AL. (2019): «¿Vecinos de toda la vida? Nimby, ocio nocturno y desapropiación en centros urbanos. La Latina, en Madrid», Athenea Digital, vol. 19 (1), p. 1-27.

REUTERS INSTITUTE FOR THE STUDY OF JOURNALISM (2018): Digital News Report 2018. Oxford, University of Oxford.

SANMARTÍN SÁEZ, J. (2019): «Análisis del discurso, ideología y neologismos: 'turismofobia', 'turistización' y ‘turistificación' en el punto de mira», Círculo de Lingüística Aplicada a la Comunicación, $\mathrm{n}^{\circ}$ 78, p. 63-90.

STORS, N. y BALTES, S. (2018): «Constructing Urban Tourism Space Digitally: A Study of Airbnb Listings in Two Berlin Neighborhoods», en Actas del Congreso ACM Human-Computer Interact. 2, CSCW, 166 (noviembre de 2018), 29 pages. (https://doi. org/10.1145/3274435)

STORS, N. y KAGERMEIER, A. (2013). «Crossing the border of the tourist bubble: Touristification in Copenhagen», en Tourismus und Grenzen (Studienzur Freizeit- und Tourismus forschung, 9). Mannheim, Meta Gis, pp. 115-131.

SWALES, J. (1990): Genre Analysis. Cambridge, Cambridge University Press.

SWALES, J. (2004): Research Genres. Cambridge, Cambridge University Press.

VAN DIJK, T.A. (2010): «Discurso, conocimiento, poder y política. Hacia un análisis crítico epistémico del discurso», Revista de Investigación Lingüística, nº 13, p. 167-215. VIVES-MIRÓ, S. y RULLÁN, O. (2017): «Desposesión de vivienda por turistización: Revalorización y desplazamientos en el Centro Histórico de Palma (Mallorca)», Revista de Geografía Norte Grande, nº 67, p. 53-71. 
